\title{
The century of metamaterials
}

\author{
Sergei Tretyakov, Aalto University, Finland \\ Augustine Urbas, Air Force Research Laboratory, USA \\ Nikolay Zheludev, University of Southampton and NTU, Singapore
}

Who, at the end of the 20th century, could have predicted that the well-established field of electromagnetism would be soon shaken to its foundation and rebuilt in years to come? Who could have expected that a classical academic subject with a 150-year history would grab mass media headlines with reports of astonishing new claims? The answer is nobody; but this revolution has happened and can be encapsulated by one word - metamaterials, which was coined in the year 2000, at the dawn of the new century [1]. The research domain of metamaterials has now grown to a fully-fledged discipline in its own right. It is a convergent field of research with top talent from microwave and optical sciences, solid state physics, materials science, acoustics, mechanics, nanotechnology and high-performance computing all attracted by the subject. Since the beginning of the century some 24 thousand research papers have been written on the subject of metamaterials. Powerful research groups rapidly emerged internationally to claim priorities and landmark achievements in a burgeoning field that has enjoyed the favour of funding agencies all over the world for many years. Unsurprisingly, broad interest in and recognition of the new discipline has created multiple parallel histories of metamaterials and diverse views on the genesis and prehistory of the discipline.

The Journal of Optics has put together this special issue on the History of Metamaterials with the aim of giving leading researchers in the field an opportunity to narrate their personals stories of engagement in metamaterials research, which as an ensemble of contributions defines the discipline as we know it today. In coordination with the publisher we furnished invitations to researchers who have accumulated the highest h-indices on the topic of "metamaterial(s)" as recorded by Web of Science, and also approached some key figures who in our view were instrumental in developing the field. To our great satisfaction the overwhelming majority of invitations were accepted, however a few groups have been unable to contribute. In particular, Prof Sir John Pendry is regrettably among these, but we are delighted that Prof Will Stewart here provides a memoir on the early days of their joint metamaterials research. Nevertheless, the Special Issue has captured the metamaterials story very well. As Guest Editors we formulated strict conditions on papers for the special issue, which we intend future historians to view as a credible source. We asked authors to be factual and chronological, and to refrain from claims of priority - the facts shall speak for themselves. Moreover, in contrast to the convention for research articles, we asked that the overwhelming majority of references should be self-citations, to prevent individual histories from becoming perhaps biased reviews papers.

So what are metamaterials? If the first paper to use the term, a "metamaterial" is defined very specifically as a "composite medium, based on a periodic array of interspaced conducting nonmagnetic split ring resonators and continuous wires"[1]. Later definitions have broadened progressively to account for the expanding meaning of the term: at around 2005 metamaterials were defined as "an arrangement of artificial structural elements, designed to achieve advantageous and unusual electromagnetic properties" [2]; in 2010, as "artificial media structured on a size scale smaller than the wavelength of external stimuli"[3]; in 2015, as "manmade media providing electromagnetic properties on-demand"[4]. Today the field rapidly expands beyond electromagnetics, and metamaterials are now seen by some as "engineered material platforms for novel wave phenomena"[5]. 
Metamaterials are now recognized as an enabling technology and one of the most buoyant and exciting research disciplines at the crossroads between photonics and nanoscience. With all the remarkable success and impact of the metamaterial research programmes internationally, the emergence of a mighty and highly skilled research community, and the substantial funding across the globe, why we do not see massive deployment of metamaterials yet? Many ask this question. There are several reasons one would need to take into consideration when answering it. First, we need to admit that the field was partially discredited by overstated promises and hype in the early days of the discipline, and we all have to take responsibility for this. This has made the industry overcautions in embracing metamaterials. Second, for a few years, due to enormous popular interest in these applications, metamaterials were seen as only relevant to superresolution and cloaking, which limited the imagination of the general public and technologists, overshadowing the potential practical impact of metamaterials in other applications. This perception is rapidly changing, but it will take time for industrial developers to appreciate the true potential of metamaterials across a wide range of technologies. Third, the field of metamaterials is not a technology as such, but a technology enabling design philosophy, and thus its proliferation faces a number of challenges: greater education of end-users and exploration required in many application fields; the development of high-throughput, cost-effective fabrication and design processes. Most importantly, however, the development of integration approaches, such as metamaterial + fibre technology, metamaterial + silicon photonic technology, and metamaterial-enabled laser and photovoltaic technologies is crucial. And finally, more time is needed: from the discovery of the light emitting diode in 1925 and LED-based optical telecommunication in 1927[6] more than 60 years was required for the massive deployment of these optical telecommunications seen currently. While pre-cursors of metamaterial research can be traced back to more than a century (e.g. [7]), the field of metamaterials is only 16 years old, and while a young field, we are already observing the promising application development.

The Special Issue editors would like to thank all contributing authors to the issue and Dr Jarlath McKenna, the IOP Journal of Optics publisher for making this special issue possible.

\section{References}

1. Smith, D.R., et al., Composite medium with simultaneously negative permeability and permittivity. Physical Review Letters, 2000. 84(18): p. 4184-4187.

2. $\quad$ http://www.metamorphose-vi.org/index.php/metamaterials

3. Zheludev, N.I., The Road Ahead for Metamaterials. Science, 2010. 328(5978): p. 582-583.

4. Zheludev, N.I., Obtaining optical properties on demand. Science, 2015. 348(6238): p. 973974.

5. $\quad$ http://congress2017. metamorphose-vi.org/

6. Zheludev, N., The life and times of the LED - a 100-year history. Nature Photonics, 2007. 1(4): p. 189-192.

7. S. Tretyakov, A personal view on the origins and developments of the metamaterial concept, Journal of Optics, 2017. 19, p. 013002. 